\title{
NEAT AND NEAT-HIGH EXTENSIONS
}

\author{
ASIF MASHHOOD, MUSHEER AHMAD AND SEEMA JAVES
}

\begin{abstract}
In the present paper, we find conditions that change a neathigh subgroup into a pure-high subgroup and a neat subgroup, to a pure subgroup. It is shown that a neat-high extension can be transformed into a pure-high extension and a neat extension, to a pure extension. Furthermore, splitting conditions for a neat exact sequence are obtained.
\end{abstract}

\section{Introduction}

A subgroup $A$ of a group $G$ is called pure in $G$ if $n A=A \cap n G$, for all natural numbers $n$. This concept was generalized by Honda [4]. He defined, a subgroup $A$ of a group $G$ is neat in $G$, if $p A=A \cap p G$, for all prime numbers $p$. This is equivalent to $m A=A \cap m G$, for all square-free natural numbers $m$. Every pure subgroup of a group $G$ is neat in $G$, but the converse is not always true. Every direct summand of a group $G$ is pure in $G$ and also neat in $G$. A sequence $O \rightarrow A \stackrel{f}{\rightarrow} G \rightarrow C \rightarrow O$ is called pure exact if $f(A)$ is a pure subgroup of $G$ and neat exact if $f(A)$ is a neat subgroup of $G$. Let $B$ be a subgroup of $G$, the exact sequence $O \rightarrow A \rightarrow G \rightarrow C \rightarrow O$ is called a $B$-pure-high extension, if $A$ is maximal disjoint from $B$ and $(A+B) / B$ is pure in $G / B$ and a $B$-neat-high extension, if $A$ is maximal disjoint from $B$ and $(A+B) / B$ is neat in $G / B$. All groups considered in this paper are abelian.

In grneral, we adopt the notations used in [2].

Received February 20, 1990. 
2. Main Results

First we find conditions for a $B$-neat-high subgroup of a $p$-group $G$ to become a $B$-pure-high subgroup of $G$. In this direction we prove the following:

Theorem 1. If $A$ is a $B$-neat-high subgroup of a p-group $G$ such that $G / B=H / B \oplus K / B$, where $H / B$ is a direct sum of cyclic groups of order $p^{n}$ and $K / B$ is a direct sum of cyclic groups of order $p^{n+1}$, then $A$ is a $B$-pure-high subgroup of $G$.

Proof. Let $\left\{H_{i} / B: i \in I\right\}$ denote the cyclic summands of $H / B$ having order $p^{n}$ and $\left\{K_{j} / B: j \in J\right\}$, those of $K / B$ having order $p^{n+1}$. It is clear that for $K \geq n+1, p^{k}(G / B)=0$ and for such $K$,

$$
p^{k}(G / B) \cap(A+B) / B=p^{k}((A+B) / B)
$$

Thus we now assume that

$$
p^{m}(G / B) \cap(A+B) / B=p^{m}((A+B) / B)
$$

for some $m$, such that $1 \leq m<n$. We prove that it holds for $p^{m+1}$ also, for this purpose let $p^{m+1}(x+B \in(A+B) / B$. for some $(x+B) \in G / B$. It follows that $p^{m+1}(x+B)=\left(a^{\prime}+B\right)$ for some $\left(a^{\prime}+B\right) \in(A+B) / B$. But by assumption, for $p^{m}(g+B)=\left(a^{\prime}+B\right),(g+B) \in G / B$ there exists an element $(a+B) \in(A+B) / B$ such that $p^{m}(a+B)=\left(a^{\prime}+B\right)$. Thus

$$
p^{m+1}(x+B)=p^{m}(a+B)
$$

$\Rightarrow p^{m}(p(x+B)-(a+B))=0 \Rightarrow(p(x+B)-(a+B)) \in G / B\left[p^{m}\right]$

If $\left(x_{i}+B\right)$ and $\left(a_{i}+B\right)$ denote the $i^{\text {th }}$ coordinates of $(X+B)$ and $(a+$ $B$ ) respectively, then it follows that $\left(p\left(x_{i}+B\right)-\left(a_{i}+B\right)\right) \in I_{i} / B\left[p^{m}\right]=$ $p^{n-m}\left(H_{i} / B\right) \subseteq p\left(H_{i} / B\right) \Rightarrow\left(a_{i}+B\right) \in p\left(H_{i} / B\right)$, for each $i \in I$. Similarly, $\left(a_{j}+B\right) \in p\left(K_{j} / B\right)$ for each $j \in J$. Consequently, there exists $(a+B) \in p(G / B)$, let $(a+B)=p(g+B)$, for $(g+B) \in G / B$. By neatness of $(A+B) / B$ in $G / B$, we 
are guaranteed an element $\left(a^{\prime}+B\right) \in(A+B) / B$ satisfying $p(g+B)=(a+B)=$ $p\left(a^{\prime}+B\right)$. But then (1) gives

$$
p^{m+1}(x+B)=p^{m}(a+B)=p^{m+1}\left(a^{\prime}+B\right) \in p^{m+1}((A+B) / B)
$$

Thus $(A+B) / B$ is pure-high in $G / B$ and $A$ is a $B$-pure-high subgroup of $G$.

In case of neat subgroups we have the following.

Theorem 2. If $A$ is a neat subgroup of a p-group $G$ such that $G=H \oplus K$, where $H$ is a direct sum of cyclic groups of order $p^{n}$ and $K$ is a direct sum of cyclic groups of order $p^{n+1}$, then $A$ is a pure subgroup of $G$.

Proof. The proof runs on similar lines as that of theorem 1.

Next, we find conditions under which the neatness of $(A+B) / B$ in $G / B$ reduces to purity of $(A+B) / B$ in $G / B$.

Lemma 1 . If $n$ is a square-free natural number and $A$ and $B$ are subgroups of $G$ such that $n(A+B)=0$, then $(A+B) / B$ is neat in $G / B$ if and only if $(A+B) / B$ is a direct summand of $G / B$.

Proof. The factor group $\frac{G / B}{\langle(A+B) / B, n(G / B)\rangle}$ is bounded and hence by theorem 17.2 of [2] is a direct sum of cyclic groups. Let $\frac{G / B}{\langle(A+B) / B, n(G / B)\rangle}=\oplus_{i \in S}<$ $\left.\bar{x}_{i}\right\rangle$, where $S$ is the set of all square-free natural numbers and $\left\langle\bar{x}_{i}\right\rangle$ is cyclic of order $n_{i}$. Define the natural homomorphism $f: G / B \rightarrow \oplus<\bar{x}_{i}>$ in such a way that for each $i \in S$ we choose $\left(x_{i}+B\right) \in G / B$ so that ${ }^{i} f\left(x_{i}+B\right)=\bar{x}_{i}$, then

$$
f\left(n_{i}\left(x_{i}+B\right)\right)=n_{i} \bar{x}_{i}=0 \Rightarrow n_{i}\left(x_{i}+B\right) \in<(A+B) / B, n(G / B)>
$$

Let $n_{i}\left(x_{i}+B\right)=\left(a_{i}+B\right)+n\left(g_{i}+B\right)$, for some $\left(a_{i}+B\right) \in(A+B) / B$ and $\left(g_{i}+B\right) \in G / B$. Since $n \bar{x}_{i}=0$, it follows that $n_{i}$ divides $n$ and hence $n_{1}$ is itself square-free for all $i$. Now,

$$
\left(a_{i}+B\right)=n_{i}\left(\left(x_{i}+B\right)-n / n_{i}\left(g_{i}+B\right)\right)
$$


Neatness of $(A+B) / B$ in $G / B$ implies that there exists $\left(a_{i}^{\prime}+B\right) \in(A+B) / B$ such that $n_{i}\left(a_{i}^{\prime}+B\right)=a_{i}+B$. If we set $y_{i}+B=\left(x_{i}+B\right)-\left(a_{i}^{\prime}+B\right)$, then with the help of relation (1)

$$
n_{i}\left(y_{i}+B\right)=\left(a_{i}+B\right)+n\left(g_{i}+B\right)-n_{i}\left(a_{i}^{\prime}+B\right)=n\left(g_{i}+B\right)
$$

Furthermore, $f\left(y_{i}+B\right)=f\left(x_{i}+B\right)=\bar{x}_{i}$

Define, $L=\left\langle n(G / B), \ldots, y_{i}+B \ldots\right\rangle$. We prove that $G / B=(A+B) / B \oplus$ $L$.

If $x \in(A+B) / B \cap L$, then

$$
x=\sum_{i \in S} m_{i}\left(y_{i}+B\right)+(n g+B) \in(A+B) / B
$$

and with the help of relation (2), $f(x)=\sum_{i \in S} m_{i} \bar{x}_{i}=0$ implies $n_{i}$ divides $m_{i}$. Now,

$$
\begin{aligned}
& n_{i}\left(y_{i}+B\right)=n\left(g_{i}+B\right) \in n(G / B) \Rightarrow m_{i}\left(y_{i}+B\right) \in n(G / B) . \\
\Rightarrow & x \in n(G / B) . \text { Consequently, } \\
& x \in(A+B) / B \cap n(G / B)=n((A+B) / B)=0
\end{aligned}
$$

and hence $(A+B) / B \cap L=0$.

Now if $(g+B) \in G / B$, then definition of $f$ and relation (2) implies that

$$
\begin{aligned}
& f(g+B)=\sum_{i \in S} m_{i}^{\prime} \bar{x}_{i}=f \sum_{i \in S} m_{i}^{\prime}\left(y_{i}+B\right) \\
\Rightarrow & f\left((g+B)-\sum_{i \in S} m_{i}^{\prime}\left(y_{i}+B\right)=0\right. \\
\Rightarrow & \left((g+B)-\sum_{i \in S} m_{i}^{\prime}\left(y_{i}+B\right)\right) \in<(A+B) / B, n(G / B)> \\
\Rightarrow & (g+B)-\sum_{i \in S} m_{i}^{\prime}\left(y_{i}+B\right)=(a+B)+n\left(g^{\prime}+B\right)
\end{aligned}
$$

for some $(a+B) \in(A+B) / B$ and $\left(g^{\prime}+B\right) \in G / B$

$$
\Rightarrow(g+B) \in<(A+B) / B, L>
$$

With the help of lemma 1, the proof of the following theorem is clear. 
Theorem 3. If $n$ is a square-free natural number, a $B$-neat-high extension $0 \rightarrow A \rightarrow G \rightarrow C \rightarrow 0$ is a B-pure-high extension if $n(A+B)=0$.

If we focus our attension to neat subgroups we have an analogue of the well known theorem that a bounded subgroup of a group $G$ is pure if and only if it is a direct summand of $G$.

Lemma 2. If $n$ is a square-free natural number and $A$ a subgroup of a group $G$ such that $n A=0$, then $A$ is a neat subgroup of $G$ if and only if $A$ is a direct summand of $G$.

Proof. Follows on similar lines as that of lemma 1.

The proof of the following theorem is clear.

Theorem 4. If $n$ is a square-free natural number, then the neat extension $0 \rightarrow A \rightarrow G \rightarrow B \rightarrow 0$ splits if $n A=0$.

Theorem 5. If $n$ is a square-free natural number and $A$ is a $n G$-high subgroup of $G$, then the sequence $0 \rightarrow A \rightarrow G \rightarrow B \rightarrow 0$ is splitting neat exact.

Proof. If $A$ is a subgroup of $G$ which is maximal with respect to the property $A \cap n G=0$, then $A$ is neat in $G$, (see [3]), that is, $m A=A \cap m G$ for all square-free natural numbers $m$. In particular, if $m=n$ then $n A=0$ and theorem 4 completes the proof.

\section{References}

[1.] L. Fuchs, Abelian Groups, Pulishing House of the Hungarian Academy of Sciences, Budapest (1958).

[2.] L. Fuchs, Infinile Abelian Groups, Vol. 1, Academic Press New York and London (1970).

[3.] D. K. Harrison, J. M. Irwin, C. L. Peercy and E. A. Walker, "High Extensions of Abelian Groups", Acia Malh. Acad. Sci. Hungar, 14, pp. 319-330, 1963.

[4.] K. Honda, Realism in the Theory of Abelian Groups 1, Comm. Math. University St. Pauli 5, pp. 37-75, 1956. 